\title{
Téoros
}

Revue de recherche en tourisme

\section{Lorsque le patrimoine se fait beau, la ville est-elle plus intéressante à voir ?}

\section{Martin Drouin}

Volume 29, numéro 2, 2010

URI : https://id.erudit.org/iderudit/1024881ar

DOI : https://doi.org/10.7202/1024881ar

Aller au sommaire du numéro

Éditeur(s)

Université du Québec à Montréal

ISSN

0712-8657 (imprimé)

1923-2705 (numérique)

Découvrir la revue

Citer cette note

Drouin, M. (2010). Lorsque le patrimoine se fait beau, la ville est-elle plus intéressante à voir ? Téoros, 29(2), 147-150. https://doi.org/10.7202/1024881ar d'utilisation que vous pouvez consulter en ligne.

https://apropos.erudit.org/fr/usagers/politique-dutilisation/ 


\title{
Lorsque le patrimoine se fait beau, la ville est-elle plus intéressante à voir?
}

\author{
Martin DROUIN, Ph.D. \\ Coordonnateur, Institut du patrimoine \\ Professeur associé, Département d'études urbaines et touristiques \\ Université du Québec à Montréal \\ drouin.martin@uqam.ca
}

L'actualité urbaine montréalaise a récemment été animée par la décision du maire de l'arrondissement du PlateauMont-Royal d'interdire les panneaux publicitaires géants sur son territoire. Les réactions furent variées, les uns condamnèrent la décision au nom de la liberté alors que d'autres applaudirent devant le choix symbolique d'une telle décision. Je suivais déjà le dossier avec intérêt lorsqu'une entrevue radiophonique m'a interpellé. Je n'ai plus souvenir de la personne interviewée, mais elle affirmait, entre autres, que l'immense panneau qui occupait le toit de l'immeuble situé au coin de l'avenue du Parc et de la rue Villeneuve nuisait à l'expression du caractère patrimonial du bâtiment. Selon cette logique, la décision des autorités de l'arrondissement de démanteler l'affiche redonnerait à celui-ci le cachet qu'il avait perdu. Je connais bien ce coin de rue, j'ai habité un peu plus haut sur l'avenue du Parc. D'ailleurs, mon immeuble servait également de socle à l'une de ces publicités géantes. Le soir, lorsque je revenais à la maison, je relevais la tête et mon regard s'accrochait invariablement sur l'image du moment. Je n'ai toutefois jamais pensé la présence de ces panneaux dans les termes exprimés par l'homme de la radio. Pour moi, elle s'inscrivait dans le paysage si particulier de ce segment de rue qui s'étend entre l'avenue du Mont-Royal, qui marque la frontière avec le parc du même nom, et la voie ferrée, plus au nord. Cette artère a ce je-ne-sais-quoi qui la classe parmi les rues emblématiques de Montréal. Bordée par des immeubles à appartements de briques dont les rezde-chaussée sont à peu près tous occupés par des commerces, elle irrigue le quartier avec ses trois voies de circulation automobile; celle du milieu — singularité amusante! change de sens selon le mouvement pendulaire des horaires de travail. Une population hétérogène l'anime et lui est sincèrement attachée ${ }^{1}$. Les deux panneaux publicitaires participent à la spécificité de cette rue que mon père, qui a travaillé dans la métropole au cours des années 1950, appelle encore "Park Avenue ${ }^{2}$. Suite à cette affaire, une question a germé en moi : faut-il que le patrimoine soit nécessairement beau pour que la ville soit attractive?

Avec ce questionnement, j'ai commencé à penser à la ville qui se transforme depuis plusieurs années sous mes yeux. D'autres interrogations se mirent alors à se succéder dans ma tête. D'abord, que vient voir le touriste urbain? Je laisserai à d'autres le soin de proposer une réponse plus élaborée, mais je noterai simplement que le visiteur recherche généralement une ville intéressante, intrigante, étonnante, fascinante, stupéfiante, singulière ou exceptionnelle comme les stratégies marketing le laissent généralement entendre. Si le degré de chaque sentiment peut varier, il n'en demeure pas moins que le choix du déplacement est habituellement influencé par le désir de goûter ce qui n'existe pas chez soi et ce qui ne se trouve pas dans une autre ville. En ce sens, c'est la somme et le dosage des ingrédients propres à chaque lieu qui participent à la qualité d'une ville. Une fois cette prémisse bien générale admise, j'ai pensé aux aménagements récemment proposés et à la manière dont le patrimoine bâti était traité. Le défi est important pour une destination touristique, car le patrimoine peut jouer un rôle fondamental comme vecteur de l'identité urbaine. Ainsi, la décision de bannir les panneaux publicitaires de l'arrondissement du Plateau-Mont-Royal découle de la volonté de créer un meilleur (et un plus beau) cadre de vie. Comme l'affirmait l'homme de la radio, le geste serait donc un pas vers ce but en valorisant, pour y arriver, la composante patrimoniale. Dans ce contexte, est-ce que la ville sera plus intéressante à voir?

On a longtemps parlé de muséification pour désigner la pratique qui favorise une mise en valeur du patrimoine axé sur le tourisme. Les opérations de requalification instrumentalisent alors le patrimoine pour en faire une sorte de décor scénographié pour le plaisir des visiteurs. La création de services vise les besoins de ces derniers que ce soit en matière d'hébergement, de restauration et d'activités commerciales. 


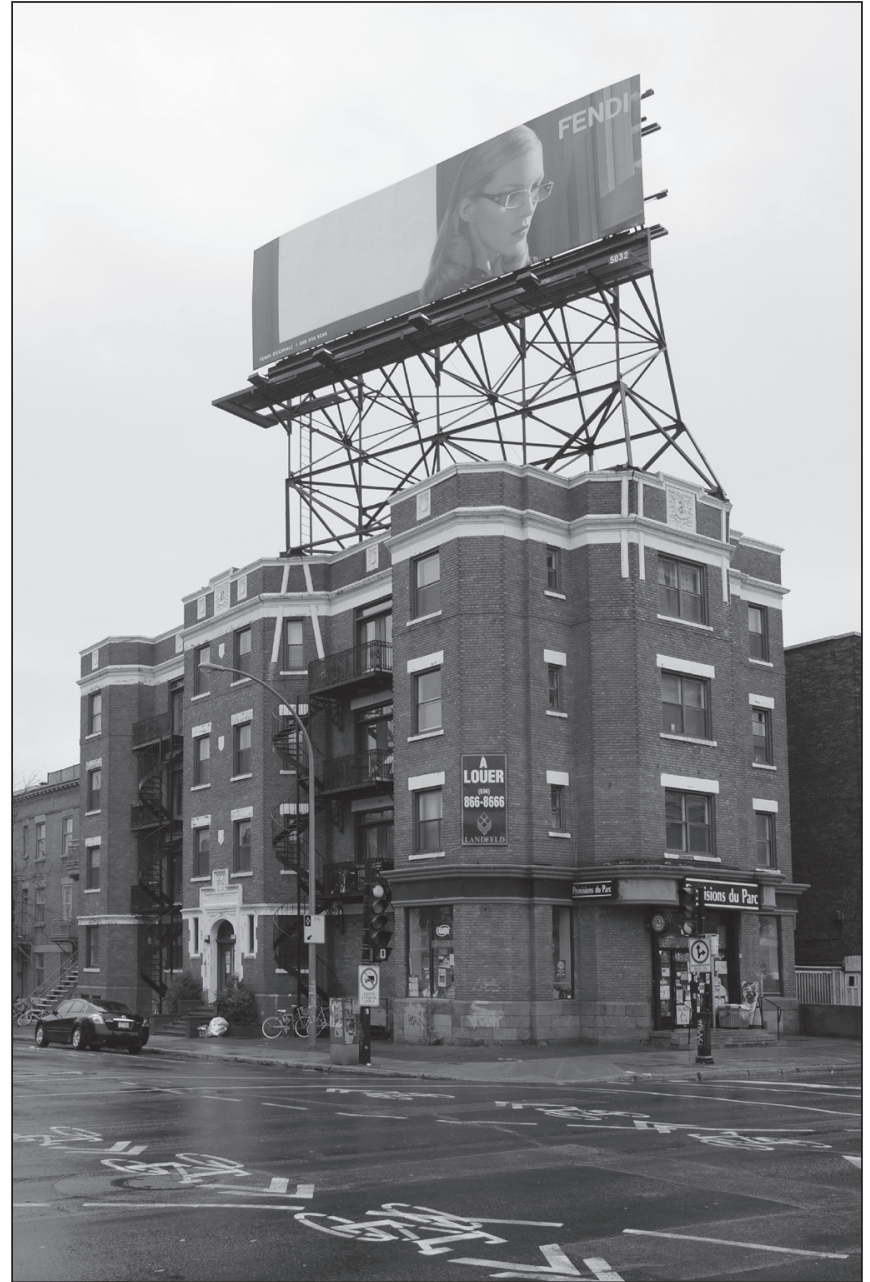

ILLUSTRATION 1 : Immeuble Pierrefonds (photo : Guillaume St-Jean).

La muséification est généralement associée à la folklorisation. La notion renvoie ici à l'image projetée par le cadre bâti ainsi aménagé. Celle-ci, souvent limitative ou réductrice, valorise une vision de la culture stéréotypée et superficielle. La rénovation des centres anciens, arrondissements historiques et autres quartiers patrimoniaux a longtemps été inscrite dans une telle dynamique. Des protestations et des remises en question ont depuis changé les philosophies d'intervention. En effet, la peur de faire de ces quartiers des lieux aseptisés, vides et sans vie, a transformé les manières de faire. Il est aujourd'hui proposé des projets de développement orientés vers le partage de l'espace entre différentes populations (résidents, travailleurs, citadins et touristes). Nous retrouvons ce "cadre de vie» que les autorités — du moins certaines - s'attachent à valoriser. Est-il possible qu'une incidence fâcheuse altère la volonté derrière le geste? Est-il possible que le désir de faire «beau " guide des choix de mise en valeur? Sans nullement vouloir faire un plaidoyer pour la sauvegarde d'affiches géantes à intégrer dans la grande famille du patrimoine urbain, pourrait-on croire que le cachet perdu et retrouvé de l'immeuble de l'avenue du
Parc s'inscrive dans cette logique? Le bâtiment est toujours le même, avec ou sans panneau publicitaire. Qu'est-ce donc qui en altère le caractère patrimonial ? L'immense structure sur le toit, me direz-vous! Certes, mais seulement dans la mesure où l'on juge négativement la valeur esthétique de la structure. Ainsi, plutôt que muséification et folklorisation, ne faudrait-il pas parler de l'esthétisation du patrimoine et, par extension, de la ville qui l'abrite?

Le patrimoine industriel est un bon exemple pour aborder cette problématique. Les villes d'Occident ont été marquées par ce que les historiens appellent la Révolution industrielle. Montréal ne fait pas exception à la règle. Au contraire, la ville, qui a bénéficié du développement économique dû à la mécanisation du travail et au labeur des ouvriers pour s'imposer pendant longtemps comme la métropole économique du Canada, s'est fortement urbanisée pendant cette période charnière de l'histoire moderne (Linteau, $2000: 15$ ). Parmi les éléments les plus remarquables, le port de Montréal et le canal Lachine s'imposent assurément. La mise en place d'une zone portuaire d'une ville digne de ce nom fut l'un des gestes lourds de conséquences posés par l'élite politique du $19^{\text {e }}$ siècle (Hanna, 1998 : 49). Quant au creusement du canal reliant Montréal à Lachine, il s'agit d'un vieux rêve qui se concrétisa avec l'ouverture à la navigation en 1825 . L'ouvrage d'ingénierie serait déjà une grande réalisation en soi s'il n'avait attiré, de surcroît, usines et entreprises pour devenir le «berceau de l'industrie manufacturière au Canada", selon l'expression de la Commission des lieux et monuments historiques du Canada qui en fit un lieu d'intérêt national dès 1929 (Chenier, 2004:112). Touchés par les transformations structurelles des villes de la seconde moitié du $20^{\mathrm{e}}$ siècle, les deux secteurs en déclin ont été au cœur d'opérations de renouveau urbain depuis les années 1970. Qu'est-il possible de visiter aujourd'hui? Peut-on encore se faire une idée de l'activité qui y régnait il y a cent ans? Il reste bien sûr des vestiges, même si beaucoup de structures ont été démantelées. Les lieux ont été aménagés avec soin, sans vouloir exagérer les gestes d'intervention. Un fait cependant s'impose : ces sites sont systématiquement devenus beaux et agréables à fréquenter. Ce n'est pas pour rien qu'une foule s'y presse pour flâner, marcher ou se balader à vélo. Que reste-t-il de la mémoire des lieux? Comme bien des auteurs l'ont souligné, le passé industriel peut difficilement être transmis dans des endroits ordonnancés, structurés, gazonnés, policés (Andrieux, 1992). On passe rapidement à une esthétique industrielle qui "oublie» de témoigner pour se concentrer sur l'aspect visuel du patrimoine, la chose à voir, non à comprendre. Ne revient-on pas aux mêmes critiques qui ont été faites il n'y a pas si longtemps à propos de la muséification et de la folklorisation?

Que doit refléter le patrimoine? Bien sûr, un bâtiment ancien n'a pas la même fonction qu'un livre d'histoire. Dès lors, est-il seulement un décor qui crée une ambiance urbaine? Entre le port de Montréal et le canal Lachine, que deviendra le silo $\mathrm{n}^{\circ} 5$ une fois réaménagé par la Société immobilière du Canada (Société immobilière du Canada, 2010)? À n'en pas douter, il frappera encore l'imaginaire du visiteur. Comment sera traitée cette immense structure 
ILLUSTRATION 2 : Rue de Bleury vers le nord, près de la rue de La Gauchetière, W. S. Keith, 26 mai 1921 (détail) (source : Archives de la Ville de Montréal, VM98,SY,D1,P011).

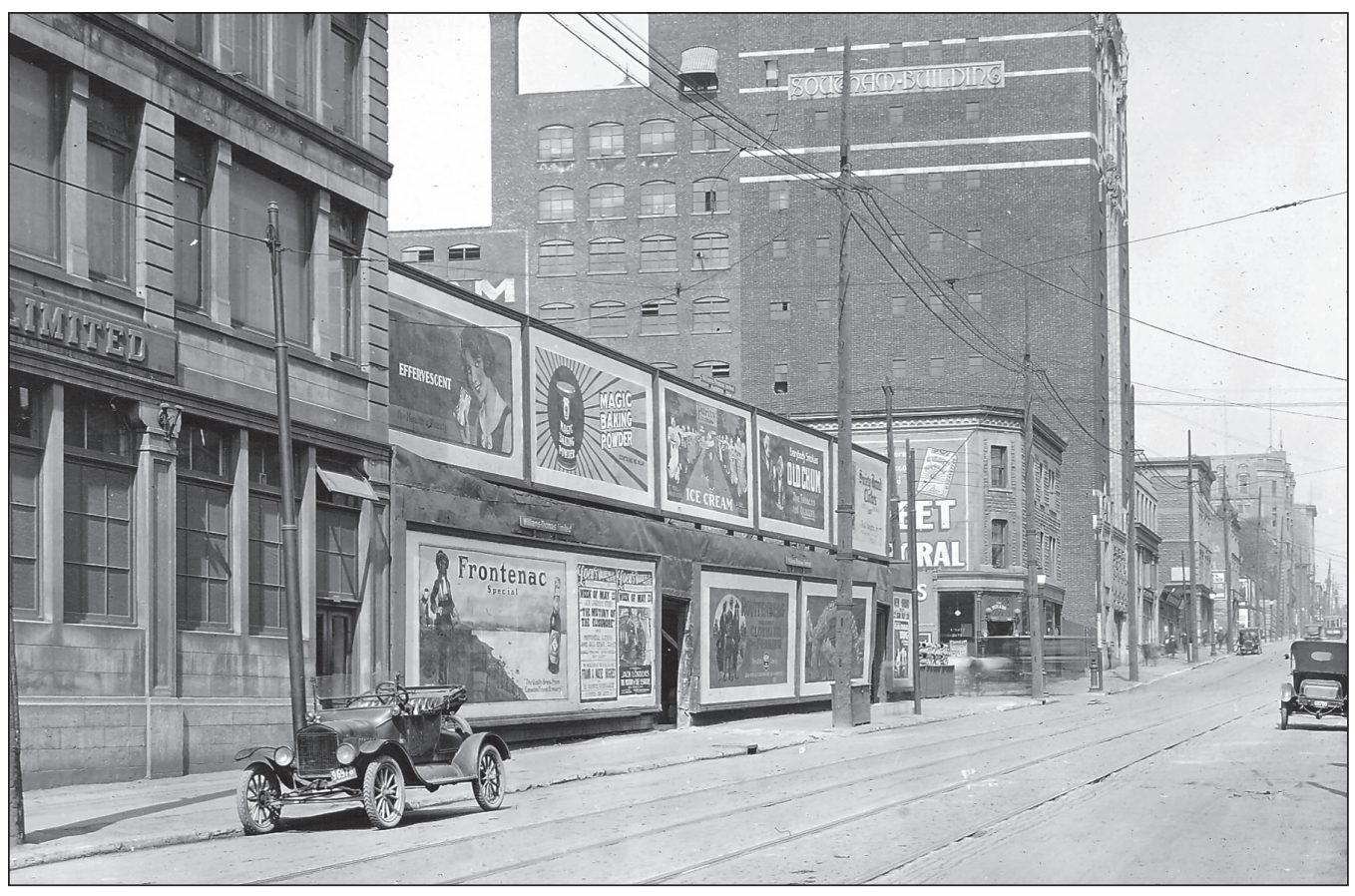

de béton que plusieurs trouvent si laide? Comprenez-moi bien, je ne suggère pas de cultiver ou d'encourager la laideur dans les villes sous prétexte d'une plus grande authenticité du patrimoine. Je me questionne simplement sur les gestes posés au nom de la mise en valeur du patrimoine. Le démantèlement du panneau publicitaire de l'avenue du Parc sera-t-il vraiment un choix positif pour affirmer le caractère patrimonial de l'édifice qui l'abritait? Assurément, l'œil du citadin ne sera plus détourné vers de futiles perspectives. L'immeuble redeviendra un immeuble à appartements construit au début du $20^{\mathrm{e}}$ siècle. C'est déjà beaucoup et, peutêtre, suffisant. Toutefois, l'avenue du Parc perdra sûrement l'un de ses repères. Bien sûr, la ville bouge, se transforme et se métamorphosera encore. On ne regrette pas la profusion des enseignes murales, peintes ou lumineuses, du Montréal des années 1920 (Archives de Montréal, 2010). Pourtant, je ne peux m'empêcher de croire que la ville, qui gomme peu à peu ses aspérités pour tenter de devenir une ville belle et soignée, devient moins intéressante.

Plusieurs exemples se sont succédés à Montréal ces dernières années. Dans la foulée du réaménagement du YMCA du centre-ville de Montréal, l'immeuble qui enjambait littéralement le boulevard Maisonneuve a été démoli (Delgado, 2000). Le passage sous le Drummond Court n'était peutêtre pas sécuritaire et invitant pour les piétons, mais ce flot d'automobiles qui transitait depuis 1957 sous la vieille conciergerie avait quelque chose de paradoxal. De plus, l'immeuble fermait la perspective du boulevard Maisonneuve pour créer une scène du paysage urbain des plus intrigantes. Non loin, sur la rue Sainte-Catherine, les travaux de rénovation de l'église unie Saint-James permirent de dégager la façade cachée par des édifices locatifs (Cardinal, 2004). La communauté religieuse avait en effet choisi de lotir le terrain en bordure de la rue Sainte-Catherine pour contrer des problèmes économiques. Depuis les années 1930, seuls les deux clochers dépassaient derrière un immeuble de briques brunes. Une enseigne en néon annonçait alors la présence de l'église Saint-James. Dans les deux cas, peu de personnes regretteront les dernières transformations. La ville semble en effet plus jolie avec la disparition de ces deux incongruités. Ces aménagements n'étaient peut-être plus admissibles pour le $21^{\mathrm{e}}$ siècle naissant, tout comme la présence des panneaux publicitaires géants sur l'avenue du Parc, mais il reste à démontrer que, en tant que curiosité urbaine, ils apportaient une complexité à l'expérience de la ville qui peut dépasser des choix apparaissant a priori plus logiques.

Pendant ce temps, paradoxalement des lieux autrement plus importants sont démolis dans l'indifférence ou, tout du moins, sans que personne ne puisse arrêter les travaux. Le cinéma Séville, protégé par les autorités municipales au début des années 1990, a été laissé à l'abandon pendant de nombreuses années au point de ne plus pouvoir en récupérer la vieille structure. Des habitations en copropriété perpétueront le nom sur le site qu'il occupait. Quel sera l'avenir de l'ancien cinéma Empress? Rien n'est définitif, mais l'avenir est encore mal assuré. Dans ce contexte, la question des panneaux publicitaires me paraît un peu vaine. Ce débat me fait penser à celui qui a eu cours au milieu des années 1990 contre l'ouverture d'une chaîne de restauration rapide près du mont Royal. Le plaidoyer des défenseurs de la montagne faisait valoir que la présence d'un restaurant aux arches dorées allait nuire au caractère patrimonial du lieu en plus de le polluer avec la présence d'emballages. Toute l'énergie de ce combat aurait pu être canalisée pour rendre l'ouverture du restaurant plus acceptable dans sa cohabitation avec le patrimoine. Si le restaurant a tout de même ouvert, il dut 


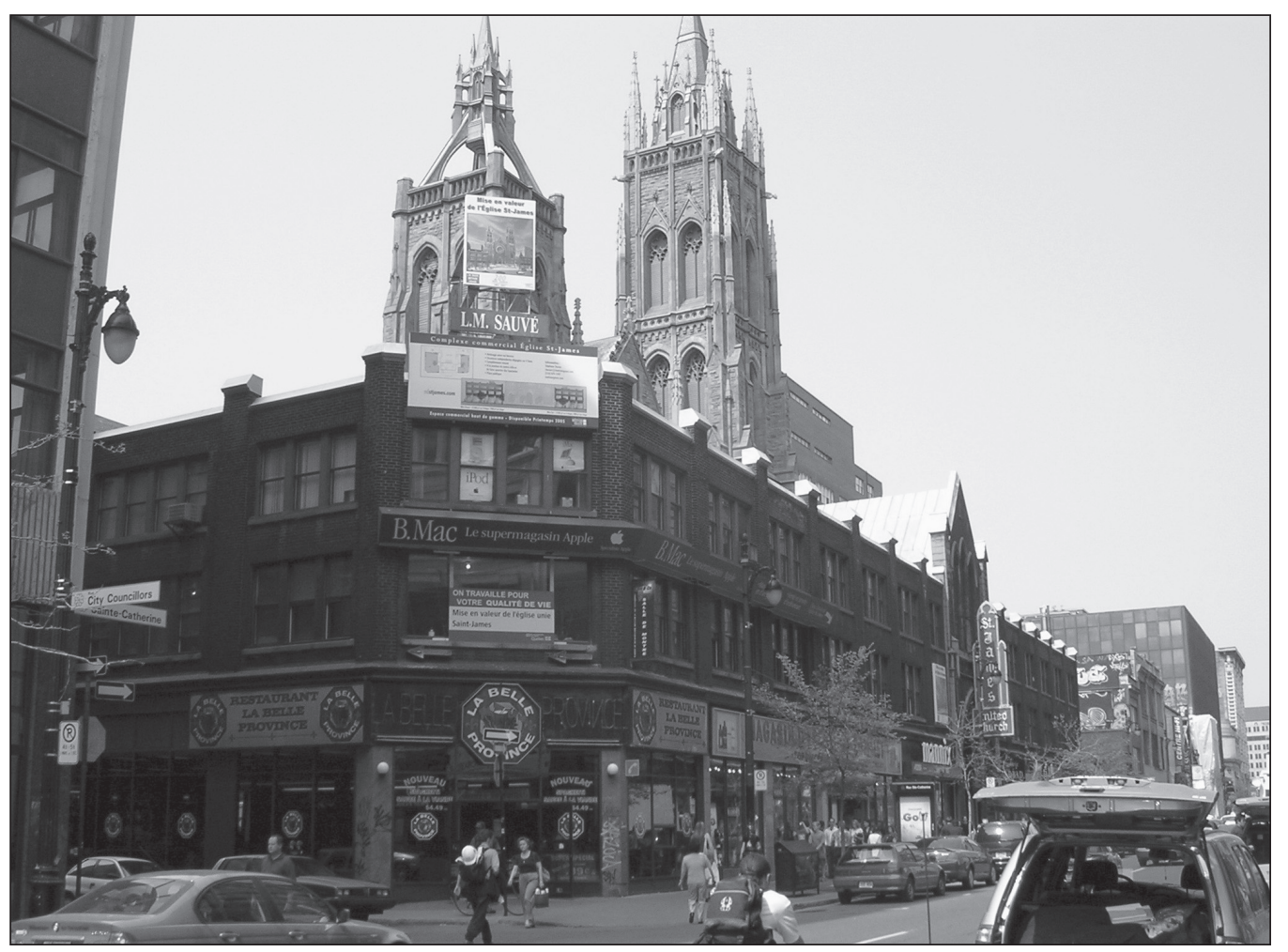

ILLUSTRATION 3 : Église unie St. James (photo : Martin Drouin). fermer ses portes une quinzaine d'années plus tard. Et la montagne est toujours là! Je comprends le raisonnement derrière l'intervention, mais ne serait-il pas mieux, comme dans le cas des panneaux publicitaires, d'utiliser les revenus d'affichage (même s'ils sont modestes) pour financer la protection du patrimoine? Ce serait peut-être un geste plus performant pour la mise en valeur du cadre de vie de l'arrondissement du Plateau-Mont-Royal. Quant à l'identité urbaine de Montréal, celle désormais présentée sous les traits de la diversité (Drouin, 2005) et que des auteurs avaient qualifiée de bric-à-brac architectural (Boivin et Comeau, 1992 : 11), ne devrait-on pas chercher à contrôler et à encourager cette délicieuse insoumission? Nous y retrouverons certainement une ville plus attractive et un patrimoine plus sensible à témoigner intelligemment d'un passé encore présent dans notre environnement urbain.

\section{Notes}

1 Sous la pression des associations de sauvegarde et de l'opinion publique, le maire Gérald Tremblay a dû retirer la demande de changement de nom de l'avenue du Parc pour l'avenue Robert-Bourassa. La mobilisation des résidents du quartier était trop importante (Drouin, 2007).

2 C'est en 1961 que le toponyme est officiellement traduit en français (Suzanne Lavigne et Nicole Rodrigue, 1995 : 367).

\section{Références}

ANDRIEUX, Jean-Yves (1992) Le patrimoine industriel, Paris : PUF. 128 p. ARCHIVES DE MONTRÉAL (2010) «Les années 1920 et les panneaux publicitaires à Montréal», Des archives plein la toile, $<\mathrm{http} / / /$ archivesdemontreal.wordpress.com/2010/09/08/les-annees1920-et-les-panneaux-publicitaires-a-montreal/>, consulté le 15 novembre 2010.

BOIVIN, Robert et Robert COMEAU (1992) «Éditorial : Montréal, l’oasis du Nord», dans Montréal, l'oasis du Nord, Paris : Autrement, p. 10-15.

CARDINAL, François (2004) «L'église St. James serait une beauté de 8 millions ", La Presse, 4 février, p. A12.

CHENIER, Rémi (dir.) (2004) Montréal, une ville d'histoire. Guide des lieux, des personnes et des événements d'importance historique nationale sur l'île de Montréal, Québec : Parcs Canada. 142 p.

DELGADO, Jérôme (2000) «Le YMCA déménage», La Presse, 13 novembre, p. B7.

DROUIN, Martin (2005) Le combat du patrimoine à Montréal (19732003), Sainte-Foy (Québec) : Presses de l’Université du Québec. 386 p.

DROUIN, Martin (2007) «Sauver le patrimoine par l'action associative : oui, mais peut-elle proposer un produit touristique de qualité?», Téoros, vol. 26, n 1, p. 67-70.

HANNA, David B. (1998) «L'importance des infrastructures de transport », DANS Montréal métropole, 1880-1930, Isabelle GOURNAY et France VANLAETHEM (dir.), Montréal : Boréal / CCA, p. 49-61.

LAVIGNE, Suzanne et Nicole RODRIGUE (dir.) (1995) Les rues de Montréal. Répertoire historique, Montréal : Méridiens/Ville de Montréal. 547 p.

LINTEAU, Paul-André (2000) Histoire de Montréal depuis la Confédération, Montréal : Boréal. 627 p.

SOCIÉTÉ IMMOBILIÈRE DU CANADA (2010) Pointe-du-Moulin, <www.pointedumoulin.ca>, consulté le 15 novembre 2010. 